\title{
Antidepressiva besser wirksam als gedacht
}

\section{In direkten Vergleichsstudien schneiden Antidepressiva etwas besser ab als Psychotherapien. Bei indirekten Vergleichen verzerren Placebo- und Noceboeffekte das Ergebnis.}

In Studien mit Antidepressiva ergibt sich für Patienten mit leichten bis mäßigen Depressionen häufig nur ein geringer klinischer Nutzen im Vergleich zu Placebo. Daher wird bei solchen Patienten in Leitlinien primär oftmals nur eine Psychotherapie empfohlen.

Psychiater um Prof. Dr. Ulrich Hegerl aus Leipzig sehen darin jedoch eine Ungleichbehandlung, denn Placebo- und Noceboeffekte verzerrten das Ergebnis in unterschiedlicher Weise.

\section{Verblindung reduziert Differenz}

So wird die Wirksamkeit von Antidepressiva in der Regel verblindet gegen Placebo geprüft. Die Patienten wissen also nicht, ob sie eine wirksame Arznei bekommen. Das wird die Hoffnung auf eine Linderung in beiden Gruppen dämpfen, in der Placebogruppe dürfte sie dennoch höher sein als ohne Behandlung, in der Verumgruppe hingegen ge- ringer als bei einer aktiven Therapie. Das, so die Schlussfolgerung der Leipziger Wissenschaftler, könnte die Differenzen zwischen Verum und Placebo reduzieren.

Bei der Psychotherapie ist eine Verblindung nicht möglich, als Kontrollgruppen dienen daher Wartegruppen oder Patienten mit Selbsthilfeansätzen. Die Patienten in der Verumgruppe wissen also, dass sie tatsächlich eine Therapie bekommen. Bei Ihnen kann man deswegen mit einem Placeboeffekt rechnen. Auf der anderen Seite wissen auch die Patienten in der Kontrollgruppe, dass sie nicht behandelt werden, und sind vielleicht enttäuscht - hier wäre dann ein Noceboeffekt zu erwarten.

Die Wirksamkeit der Psychotherapie werde dadurch überschätzt und die Wirksamkeit der Antidepressiva in placebokontrollierten Studien unterschätzt, mutmaßen die Forscher um Hegerl. Dies konnten sie in einer eigenen Analyse bestätigen: In Studien mit einem direkten Vergleich von Psychotherapie gegen Antidepressiva schnitten letztere besser $a b$. Hatten die Studien zusätzlich Placebo und/oder Wartegruppen, lag der Vorteil hingegen bei der Psychotherapie.

European Psychiatry 2015, 30(6): 685-693.

... drängt sehr niedrig dosiertes Buprenorphin Suizidgedanken zurück. Dies könnte bei Patienten interessant sein, die auf andere Optionen kaum ansprechen.

Am J Psychiatry 2016; 173(5): 491-498.

.... scheint eine Depression das Demenzrisiko zu vervielfachen, wenn das Gehirn viel Beta-Amyloid enthält. Das hat eine PETStudie ergeben, die bei der Jahrestagung der American Academy of Neurology in Vancouver vorgestellt wurde.

68th Annual Meeting of the AAN, Vancouver, April 2016, Platform Session S35.003.

.... ist der postkoitale Blues bei Frauen gar nicht so selten. Fast jede zweite junge Frau war nach dem Sex schon mal grundlos depressiv. Bei $2 \%$ ist das regelmäßig der Fall. Darauf deutet eine Studie.

(mut)

Sexual Medicine 2015; 3(4): 235-243.

Punkte sammeln in der Sommerakademie mit e.Med

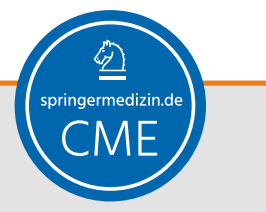

Auch 2016 haben wir in der Sommerakademie ausgewählte CME-Kurse für Ihre hausärztliche Fortbildung zusammengestellt.

Zum Thema "Depressionen" finden Sie unter www.springermedizin.de/sommerakademie aktuell den Kurs

"Körperliche Aktivität bei unipolaren Depressionen: Bewegung als evidenzbasierter Baustein der multimodalen Therapie"

\section{Verhaltenstherapie gegen Depressionen}

Ein kurzes Präventionsprogramm mit kognitiver Verhaltenstherapie (KVT) kann Depressionen bei gefährdeten Jugendlichen über viele Jahre verhindern.

In einer Studie ließ sich die Depressionsinzidenz mit spezieller KVT bei gefährdeten Jugendlichen deutlich senken. Ärzte prüften das Verfahren bei 316 Teenies mit einer ersten depressiven Episode oder subklinischen Symptomen.

Die Hälfte erhielt die übliche medizinische Versorgung, die übrigen das Präven- tionsprogramm - wöchentlich eine 90 Minuten dauernde KVT-Sitzung über zwei Monate hinweg. Die Schwerpunkte lagen auf kognitiver Restrukturierung und Problemlösestrategien. Danach konnten die Teilnehmer ein halbes Jahr lang jeden Monat an einer Auffrischsitzung teilnehmen. Über sechs Jahre hinweg erkrankten Personen der KVTGruppe signifikant seltener (-29\%) an einer Depression als Teilnehmer der Kontrollgruppe.

(mut)

JAMA Psychiatry 2015; 72(11): 1110-1118.
Zur Teilnahme benötigen Sie das e.Med-Abo, mit dem Sie Zugang zu allen Inhalten auf Springer-Medizin.de erhalten - kombiniert mit einer gedruckten Fachzeitschrift Ihrer Wahl. Als e.Med-Abonnent haben Sie nicht nur Zugriff auf die Sommerakademie, Sie können das komplette CME-Kursangebot von SpringerMedizin.de nutzen. Hier finden Sie aktuell rund 550 CME-zertifizierte Fortbildungskurse aus allen medizinischen Fachrichtungen! Damit ist CME.SpringerMedizin.de Deutschlands erste Adresse für Online-CMEFortbildung.

Nutzen Sie die Sommerzeit, um CME-Punkte zu sammeln und um sich schnell und effizient fortzubilden. Testen Sie CME.SpringerMedizin.de 30 Tage lang kostenlos und unverbindlich mit dem e.Med-Abo: www.springermedizin.de/eMed 